\title{
Arritmias cardíacas: Diagnósticos de Enfermagem baseados na Taxonomia da NANDA-I (2018-2020)
}

\author{
Cardiac arrhythmias: Nursing Diagnoses based on the NANDA-I Taxonomy (2018-2020) \\ Arritmias cardíacas: Diagnósticos de Enfermería basados en taxonomía \\ NANDA-I (2018-2020) \\ Adriana Souza Szpalher ${ }^{1 *}$, Marianne Cardoso Batalha².
}

\begin{abstract}
RESUMO
Objetivo: identificar os diagnósticos de enfermagem ao portador de arritmia cardíaca para promoção do apoio na realização do Processo de enfermagem para estes indivíduos. Métodos: revisão integrativa no período de maio a julho de 2019 nas bases de dados BVS, PubMed, SciELO, CENTRAL, CAPES, ProQuest e Google Acadêmico. O processo de análise do artigo selecionado deu-se por meio da leitura exploratória e detalhada de títulos, resumos e dos resultados das pesquisas, onde se buscaram os diagnósticos de enfermagem relacionados às arritmias. Resultados: Diagnósticos de Enfermagem identificados foram dor aguda relacionado a agentes lesivos biológicos e físicos evidenciado no relato verbal de dor e expressão facial; Insônia relacionado à ingestão de estimulantes ou álcool evidenciado com limitação do padrão normal do sono e por dificuldade de ordenar ou manter o sono; Estilo de vida sedentário relacionado a falta de motivação e de interesse evidenciado pela falta de condicionamento físico. Considerações finais: por haver escassa produção de estudos relacionados ao objetivo, sugerem-se estudos que desenvolvam o Processo de Enfermagem ao portador de arritmias nos contextos intra e extra-hospitalar. Ainda se sugere a investigação de diagnósticos em outros domínios que não foram abordados, visando, assim, a atuação de forma holística.
\end{abstract}

Palavras-Chave: Diagnóstico de Enfermagem, Arritmias cardíacas, Enfermagem Cardiovascular, Processo de Enfermagem.

\begin{abstract}
Objective: To identify nursing diagnoses for patients with cardiac arrhythmia to promote support in the realization of the Nursing process for these individuals. Methods: integrative review from May to July 2019 in the databases VHL, PubMed, SciELO, CENTRAL, CAPES, ProQuest and Google Scholar. The analysis process of the selected article took place through the exploratory and detailed reading of titles, abstracts and research results, where the nursing diagnoses related to arrhythmias were sought. Results: Nursing Diagnoses identified were acute pain related to biological and physical injurious agents evidenced in the verbal report of pain and facial expression; Insomnia related to the ingestion of stimulants or alcohol evidenced with limitation of normal sleep pattern and difficulty in ordering or maintaining sleep; Sedentary lifestyle related to lack of motivation and of interest evidenced by lack of physical fitness. Final considerations: Due to the scarce production of studies related to the objective, we suggest studies that develop the Nursing Process for patients with arrhythmias in the intra and extra-hospital contexts. It is still suggested to investigate diagnoses in other domains that were not addressed, thus aiming at acting holistically.
\end{abstract}

Key words: Nursing Diagnosis, Arrhythmias Cardiac, Cardiovascular Nursing, Nursing Process.

${ }^{1}$ Residência em Clínica e Cirúrgica Geral - Universidade Federal do Estado do Rio de Janeiro (UNIRIO), Rio de Janeiro-RJ. *E-mail: adrisz88@hotmail.com

2 Docente do Centro Universitário Celso Lisboa (UCL). Doutoranda da Escola de Enfermagem Anna Nery (EEAN) - Universidade Federal do Rio de Janeiro (UFRJ), Rio de Janeiro-RJ. 


\section{RESUMEN}

Objetivo: identificar diagnósticos de enfermería para pacientes con arritmia cardíaca para la promoción de apoyo en la realización del Proceso de enfermería para estos individuos. Métodos: revisión integradora de mayo a julio de 2019 en bases de datos BVS, PubMed, SciELO, CENTRAL, CAPES, ProQuest y Google Scholar. El proceso de análisis del artículo seleccionado se realizó a través de lectura exploratoria y detallada de títulos, resúmenes y resultados de investigación, donde se buscaron diagnósticos de enfermería relacionados con las arritmias. Resultados: Diagnósticos de Enfermería identificados fueron dolor agudo relacionado con agentes nocivos biológicos y físicos evidenciados en el informe verbal de dolor y expresión facial; El insomnio relacionado con la ingestión de estimulantes o alcohol evidenciado con limitación del patrón de sueño normal y dificultad para ordenar o mantener el sueño; El estilo de vida sedentario relacionado con la falta de motivación e de interés evidenciado por falta de aptitud física. Consideraciones finales: Debido a la escasa producción de estudios relacionados con objetivo, sugerimos estudios que desarrollen el Proceso de Enfermería para pacientes con arritmias en los contextos intra y extrahospitalarios. Todavía se sugiere investigar diagnósticos en otros dominios que no se abordaron, con el objetivo actuar de manera integral.

Palabras clave: Diagnóstico de Enfermería, Arritmias Cardíacas, Enfermería Cardiovascular, Proceso de Enfermería.

\section{INTRODUÇÃO}

As doenças cardiovasculares isquêmicas e cerebrovasculares foram as causas mais frequentes de morte no país em 2013, sendo o principal componente de 70\% das mortes em idosos (maiores de 60 anos). Assim, estas lideram a lista de óbitos em nível mundial e são as principais causas de morte no Brasil. Entre as doenças crônicas, as do aparelho circulatório suportam a maior carga de mortalidade prematura entre 30 a 69 anos de idade. Apesar de a mortalidade ter diminuído ao longo dos anos no Brasil, ela ainda continua alta. A ingestão de álcool está relacionada às cardiopatias, sendo que, sua ingesta prolongada pode levar ao desenvolvimento de fibrilação atrial (FA) ao aumento da pressão arterial sistólica (REHM J, et al., 2010).

Conforme Passinho RS, et al. (2018) as arritmias são equivalentes anginosos da isquemia do miocárdio, na quais a bradicardia é caracterizada pela diminuição do ritmo cardíaco durante um processo isquêmico, ocasionada pela estimulação do sistema parassimpático ou por um medicamento que gere este efeito; pode ocorrer durante o tratamento da dor da isquemia ao utilizar opioides ou ao utilizar betabloqueadores, este último para prevenção de arritmias. A taquicardia pode acompanhar a precordialgia e é causada pela hiperatividade do sistema simpático. Passinho RS, et al. (2018) complementam que esses novos focos causadores de arritmias se localizam em qualquer ponto do miocárdio, tanto em nível atrial quando ventricular, gerando ectopias e, assim, ocasionando impulsos elétricos anormais.

A American Heart Association (AHA), em 2018 relata que a apresentação clínica da fibrilação ventricular (FV) varia de ausência de sintomas até a parada cardio-respiratória (PCR). Dos sobreviventes, mais de 50\% das PCR em ambiente extra-hospitalar terão significantes lesões arteriais coronarianas. Luzia MF, et al. (2014) identificaram as condições cardiovasculares, como arritmias, um processo patológico associado com a ocorrência de quedas. Rienstra M, et al. (2012) pontuam que a FA é a arritmia mais comum, está associada com o aumento do risco de acidente vascular cerebral, insuficiência cardíaca, demência e morte; e corroborando com a AHA, lembram que $15 \%$ à $30 \%$ dos portadores são assintomáticos.

Assim sendo, a compreensão clínica e científica dos problemas de saúde apresentadas pelo indivíduo com arritmia, além do olhar crítico do enfermeiro se tornam importantes fatores para as ações de enfermagem a serem prestadas (ALMEIDA DV, et al., 2013). Para organizar as condutas e cuidados planejados, o enfermeiro faz uso do Processo de Enfermagem (PE), um instrumento metodológico que orienta os procedimentos de Enfermagem, qualifica o cuidado e direciona a documentação da prática profissional; é sistematizado por meio das etapas avaliação, diagnóstico, planejamento, implementação e avaliação (COFEN, 2009). 
A Taxonomia da Associação de Diagnósticos de Enfermagem da América do Norte - Internacional (NANDA-I, sigla no idioma inglês) guia a etapa de Diagnóstico de Enfermagem (DE) e determina intervenções e resultados de enfermagem. Logo, realiza-se a coleta de dados para identificar problemas de saúde/processos de vida e riscos do indivíduo e, assim, analisar e formular os potenciais DE.

Carmo TG (2018) identificou as arritmias cardíacas benignas e malignas como fatores importantes associados ao DE "Recuperação cirúrgica retardada" nas fases pré e pós-operatórias de cirurgia cardíaca; e identificou uma taxa de $9,55 \%$ de readmissão hospitalar durante os primeiros 30 dias de pós-operatório, relacionada à presença de flutter atrial e bloqueio atrioventricular. Conforme Andrade MVM, et al. (2014) o enfermeiro deve estar apto a prestar assistência frente a arritmia, baseado no quadro clínico. Assim, vê-se a importância em identificar desvios no padrão das respostas humanas para desenvolver os DE apropriados.

Nesse contexto, este estudo justifica-se pela importância e magnitude das Doenças Cardiovasculares (DCV) e particularmente das arritmias, mazelas estas que podem manifestar-se nos diversos cenários de saúde, como em hospitais e unidades de saúde básica, e no contexto social, como nas próprias ruas e no trânsito. Logo, observa-se a relevância da inclusão das arritmias, emergenciais ou não, no planejamento de cuidados e no PE. Diante disto, foram observados diversos questionamentos, originando, assim, a pergunta norteadora: Que diagnósticos são inerentes e implementáveis ao portador de arritmia cardíaca?

Com esta pesquisa, pretende-se fomentar reflexões acerca do saber-agir de enfermeiros frente as condutas necessárias ao portador de arritmia, além de proporcionar discussões acerca de estratégias para o cuidado por meio da identificação de problemas de saúde reais e potenciais inerentes a este indivíduo. Portanto, o objetivo é revisar e identificar os diagnósticos de enfermagem ao portador de arritmia cardíaca para promoção do apoio na realização do Processo de enfermagem (PE) para estes indivíduos.

\section{MÉTODOS}

Para a revisão integrativa foi realizada uma busca no período de maio a julho de 2019 nas bases de dados Biblioteca Virtual de Saúde (BVS), US National Library of Medicine (PubMed), Scientific Electronic Library Online (SciELO), The Cochrane Central Register of Controlled Trials (CENTRAL), Portal da Coordenação de Aperfeiçoamento de Pessoal de nível superior (CAPES), ProQuest Dissertations e Google Acadêmico. Tais bases são recomendadas em Diretrizes metodológicas para elaboração de revisão sistemática e metanálise de estudos comparativos sobre fatores de risco, do Ministério da Saúde (BRASIL, 2014). Assim, optou-se por realizar a revisão bibliográfica com base nas ferramentas de busca mencionadas, visando abranger literaturas americanas (do Norte e Sul) e européias, nos diversos idiomas, além de obter maior diversidade de estudos como artigos originais, teses/dissertações, revisões, livros e outros.

Os critérios para a inclusão dos artigos foram: originais ou revisão de literatura, relatos de caso (experiência), dissertações, teses, possuírem resumo disponível, acesso aberto, trabalhos apresentados em congressos, apresentarem Diagnósticos de Enfermagem (DE) para qualquer arritmia. Foram excluídos os estudos de reflexão, as cartas ao editor, artigos sobre arritmias que não trouxessem DE, artigos sobre cardiopatia que não trouxessem DE para arritmia, artigos sobre cardiopatia neonatal ou infantil com ou sem $\mathrm{DE}$. Assim, foi realizada extensa busca em bases nacionais e internacionais, triagem e análise dos artigos, conforme literatura (DONATO H e DONATO M, 2019). Para a seleção de artigos foi realizado recorte temporal no período de 2010 a 2019, intencionando-se, assim, buscar os DE mais atualizados, visto a NANDA-I realizar a revisão, alteração e exclusão frequentes na taxonomia.

\section{Seleção dos estudos}

As estratégias de busca retornaram 1111 estudos, prevalecendo o idioma inglês; houve 2 artigos duplicados. 1101 artigos foram excluídos após análise do título e resumo, 9 artigos foram analisados no seu todo e, assim, 1 atendeu ao objetivo do estudo, considerando-se os critérios de inclusão e exclusão. Decidiuse em não limitar os estudos por meio de estratos indicativos de qualidade, como o Qualis, intencionando-se resgatar a maior quantidade viável de estudos sobre a temática. 
Os artigos foram analisados por um revisor Enfermeiro com especialização e um revisor Enfermeiro doutorando, por meio dos títulos; leitura dos resumos e análise detalhada, que é leitura da introdução, metodologia, resultado e conclusão. A organização do resultado da busca foi realizada com base no diagrama do PRISMA- Preferred Reporting Items for Systematic Reviews and Meta-Analyses (Figura 1).

Figura 1 - Diagrama de seleção de artigos

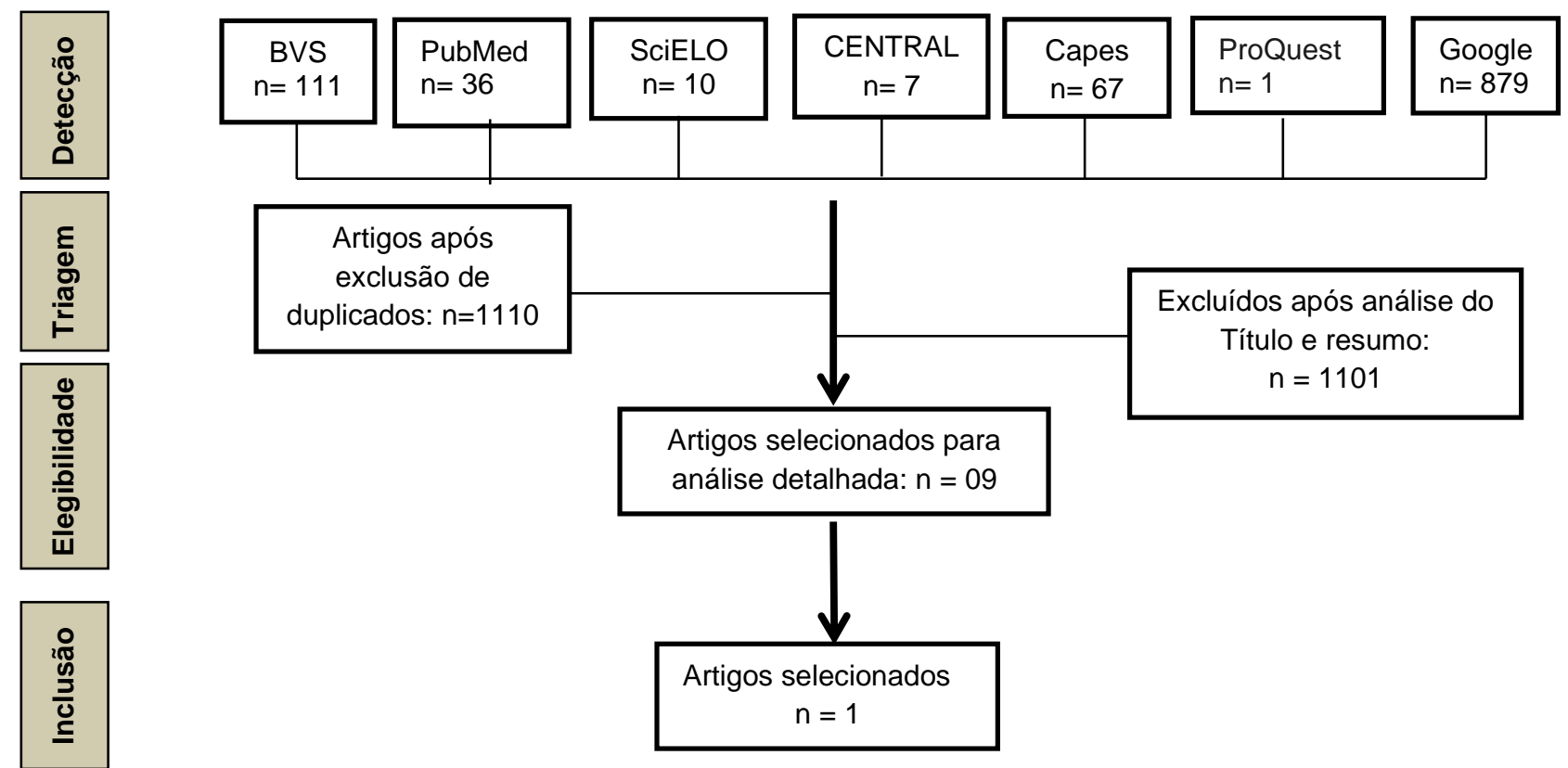

Fonte: Szpalher AS, Batalha MC, 2019.

Ressalta-se que não houve delimitação por estratos indicativos de qualidade. Foram utilizados os descritores (DeCS): "diagnóstico de enfermagem" e "arritmias cardíacas" em português, inglês e espanhol, além do termo usualmente utilizado: arritmia, combinados com os operadores booleanos "AND" e "OR". A CENTRAL também sugeriu a busca com os termos arrhythmical e arrhythmic. As expressões de busca estão apresentadas no Quadro 1.

Quadro 1 - Bases de dados e estratégias de busca utilizadas.

\begin{tabular}{|l|l|}
\hline \multirow{3}{*}{ BVS/ Lilacs } & $\begin{array}{l}\text { tw:(("Diagnóstico de Enfermagem" OR “nursing diagnosis" OR "diagnóstico } \\
\text { de enfermería") AND ("Arritmias Cardíacas" OR "arrhythmias, cardiac" OR } \\
\text { arritmia)) AND (instance:"regional") AND (year_cluster:("2012" OR "2014" } \\
\text { OR "2013" OR "2010" OR "2011" OR "2015" OR "2016" OR "2017" OR } \\
\text { "2018" OR "2019")) }\end{array}$ \\
\hline PubMed/ MedLine & ("arrhythmias, cardiac" OR Arrhythmias) AND "nursing diagnosis" \\
\hline CENTRAL & Arrhythmias, arrhythmia, arrhythmical, arrhythmic AND nursing \\
\hline Google & "diagnósticos de enfermagem" arritmia cardíaca \\
\hline Portal CAPES & $\begin{array}{l}\text { ("Diagnóstico de Enfermagem" OR "nursing diagnosis" OR "diagnóstico de } \\
\text { enfermería") AND ("Arritmias Cardíacas" OR "arrhythmias" OR arritmia) }\end{array}$ \\
\hline ProQuest & "nursing diagnosis" AND arrhythmia \\
\hline SciELO & $\begin{array}{l}\text { ("Diagnóstico de Enfermagem" OR "nursing diagnosis" OR "diagnóstico de } \\
\text { enfermería") AND ("Arritmias Cardíacas" OR "arrhythmias" OR arritmia) }\end{array}$ \\
\hline
\end{tabular}

Fonte: Szpalher AS, Batalha MC, 2019. 


\section{RESULTADOS}

Ao utilizar as estratégias de busca mencionadas, outros temas foram apresentados; a busca no portal BVS e no SciELO retornaram estudos inéditos e de revisão, relacionados à diagnósticos de enfermagem na insuficiência cardíaca, cardioversão, hipercalemia, habilidade dos enfermeiros na realização de procedimentos, epidemiologia e benefícios do cuidado coordenado nas arritmias, avaliação da qualidade de vida, fatores de risco para cardiopatias, uso de monitor multiparamétrico e outros. A insuficiência cardíaca e infarto foram cardiopatias majoritariamente abordadas. As bases nacional (CAPES) e internacional (ProQuest) não retornaram teses/dissertações condizentes com o Objetivo proposto.

O Google acadêmico retornou maior quantidade de resultados, porém não foi encontrado estudo que respondesse à questão desta pesquisa. Alguns assuntos encontrados foram: assistência de enfermagem na unidade de hemodiálise, insuficiência renal crônica e diálise, assistência às crianças com cardiopatias, ao doador de órgãos, cuidados em terapia intensiva, transplante cardíaco, estomias, sepse, transplante hepático, grande queimado, competências da enfermagem frente às situações críticas e outros, todos tendo as arritmias como comorbidades presentes. Com relação às cardiopatias, a insuficiência cardíaca foi o tema mais abordado. A PubMed apresentou, na sua maioria, artigos realizados por enfermeiros e relacionados à assistência, porém sem abordagem aos DE para arritmias.

O artigo que atendeu o objetivo proposto é um relato de caso de Gonzalez B, et al. (2016) sobre um cliente de 32 anos admitido no setor de emergência queixando-se de palpitações, tontura e precordialgia, taquicardia de ritmo regular e hipertensão arterial, diagnosticado com arritmia, apresentando os DE: dor aguda relacionado a agentes lesivos biológicos e físicos evidenciado no relato verbal de dor e expressão facial; Insônia relacionado à ingestão de estimulantes ou álcool evidenciado com limitação do padrão normal do sono e por dificuldade de ordenar ou manter o sono; estilo de vida sedentário relacionado a falta de motivação e interesse evidenciado por demonstra falta de condicionamento físico. A avaliação deve conter histórico e exame físico minuciosos, e ainda comentam sobre a importância da instrumentalização do enfermeiro por meio do PE e sua aplicação nas diferentes realidades onde este indivíduo está inserido.

Norekvål TM, et al. (2017) lembram que o enfermeiro está na vanguarda do atendimento ao portador de arritmias ou ao indivíduo com risco para desenvolvê-lo, cabendo-lhe a responsabilidade de assistir estes indivíduos e suas famílias. Almashrafi A, et al. (2016), ao estudarem fatores que prolongam a internação na Unidade de Terapia Intensiva (UTI) pós cirurgia cardíaca, apontaram como um dos principais fatores a FA/arritmia, que é considerada benigna, porém com alto potencial de complicações, podendo afetar entre $30 \%$ a $50 \%$ dos pacientes.

Como exemplo, a pesquisa descritiva e exploratória de Galvão PCC, et al. (2016), sobre admissões em emergência relacionadas à insuficiência cardíaca descompensada, identificou as arritmias $(24,4 \%)$ como segundo fator precipitante do último episódio de descompensação. Rienstra M, et al. (2012) alertam para o fenômeno mundial de envelhecimento populacional, cenário no qual se prevê aumento dramático da prevalência de FA. Nottingham F (2010) menciona o fator idade avançada ao aumento das taxas de doenças cardíacas gerais e prevê o total de 6 milhões de acometidos, somente pela FA, no ano de 2050.

Em sua pesquisa de revisão integrativa, Passinho RS, et al. (2018) identificaram a arritmia cardíaca como o 4 $4^{\mathrm{a}}$ sintoma mais comum nos indivíduos acometidos pelo infarto agudo do miocárdio (IAM). Os achados identificados, relacionados à arritmia, foram os distúrbios de condução, bloqueio atrioventricular, bradiarritmia e taquiarritmia, taquicardia (supraventricular e ventricular), FA e FV e palpitação.

Ainda lembram que a arritmia é uma complicação relevante no IAM e ressaltam a necessidade da presença da equipe de enfermagem, de modo a identificar os problemas de saúde e traçar condutas. A AHA (2018) evidencia a importância em se conhecer o quadro clínico para traçar os cuidados necessários, visto alguns sintomas serem inespecíficos. Assim, são apresentados os achados clínicos nas arritmias na Figura 2. 
Figura 2 - Caracterização das arritmias

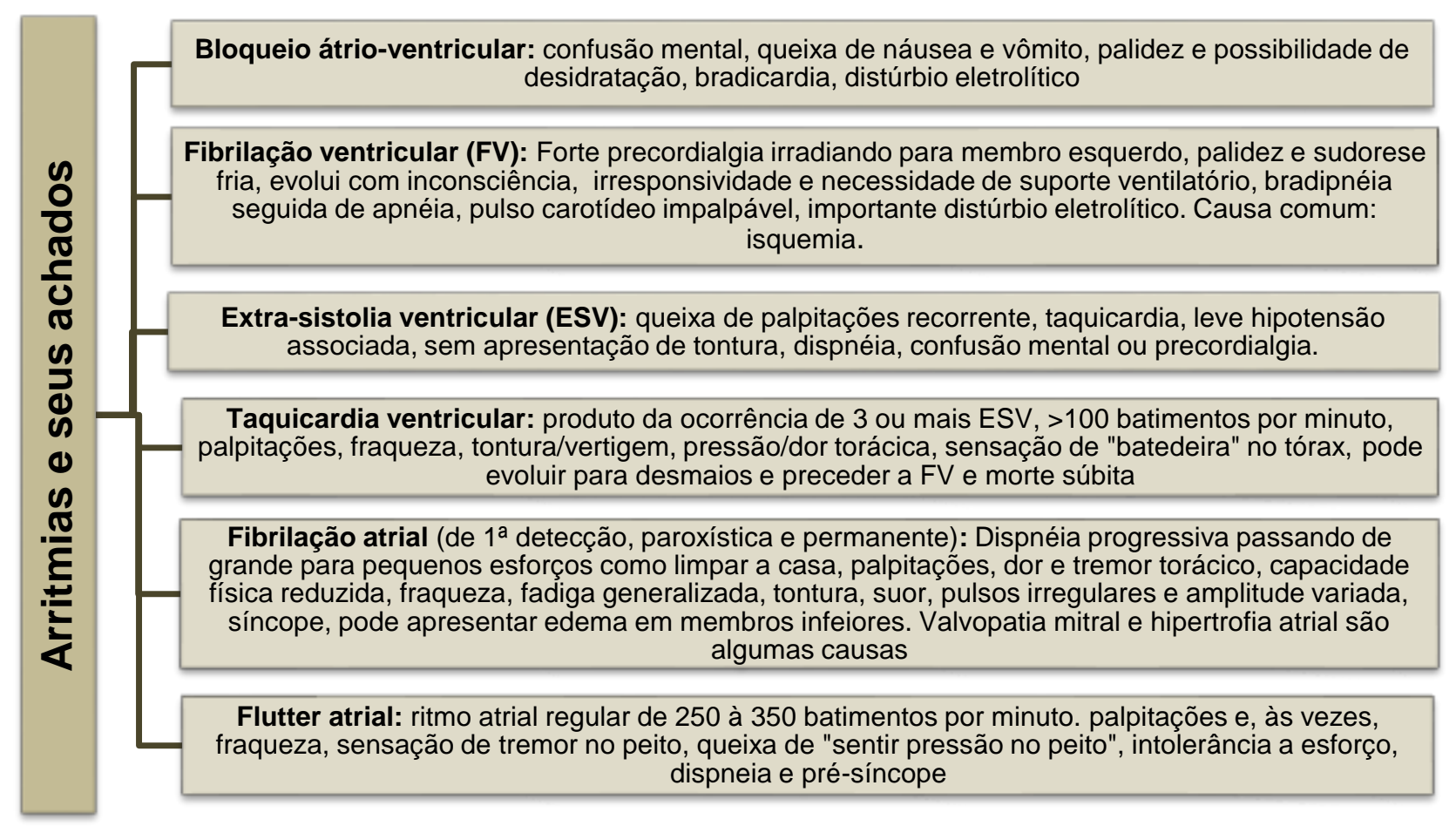

Fonte: Szpalher AS, Batalha MC, 2019.

\section{DISCUSSÃO}

Observa-se escassa produção acerca do objetivo proposto; fato este que pode estar relacionada ao desconhecimento da magnitude e da gravidade das arritmias pelo enfermeiro, por não saberem reconhecer essa falha de condução ao examinar o eletrocardiograma ou pode estar motivado pela abordagem ineficaz da temática na graduação. Andrade MVM, et al. (2014) também identificaram tal resultado em seu estudo, acrescentando que esse desconhecimento pode interferir direta e negativamente no prognóstico e no cuidado ao portador de arritmias.

Apesar de os estudos concordarem com relação aos achados clínicos das arritmias, Rienstra M, et al. (2012) lembram que há diversidade nos tipos e na severidade dos sintomas, sendo apresentados tanto de um indivíduo para o outro quanto no próprio indivíduo. Essa flutuação sintomática altamente variável ocorre ao longo do tempo, aumentando, assim, a complexidade da arritmia. Assim sendo, os DE sugeridos adiante não são imutáveis, são passíveis de alterações e devem ser utilizados para nortear o PE, tendo como base a fisiopatogia, o histórico do indivíduo, sua realidade social e particularidades no enfrentamento da doença para, segundo Nottingham (2010), fornecer alívio dos sintomas e melhorar a qualidade de vida.

A Taxonomia de NANDA-I 11ำ edição (2018-2020) apresenta 244 DE, organizados em 13 domínios, que são as áreas de interesse e são agrupados com atributos comuns por 47 classes, no qual as áreas de preocupação do Enfermeiro estão classificadas e categorizadas, para serem utilizados na prática do Cuidar de enfermagem (NANDA, 2018). Logo, o Enfermeiro trata as respostas humanas aos problemas de saúde apresentados pelo cliente, e usam as Taxonomias como auxiliador. Buscou-se abranger todos os domínios da taxonomia, de modo a cobrir todas as esferas de saúde-doença por qual o acometido por arritmias experiencia.

Adrietta MP, et al. (2011) e Galvão PCC, et al. (2016) ressaltam o papel de educador do enfermeiro, cabendo-Ihe orientações em saúde e auxílio para promover o autocuidado; e ainda, o plano de cuidados deve ser individualizado diante do contexto apresentado, tão necessárias ao indivíduo cardiopata. 
Diante da fisiopatologia e dos achados clínicos identificados na literatura para as principais arritmias, são elencados os possíveis DE aplicáveis a estes indivíduos, quer seja em ambiente hospitalar, em atendimento domiciliar, na Atenção primária ou no contexto da residência/ambiente domiciliar. Assim, totalizou-se 10 domínios, 13 classes e 27 DE reais (Quadro 2) e potenciais (Quadro 3).

Quadro 1 - DE reais sugeridos ao portador de arritmia.

\begin{tabular}{|c|c|c|}
\hline Domínios/Classes & Diagnósticos de enfermagem reais & Fatores relacionados \\
\hline $\begin{array}{l}\text { Promoção da saúde / Percepção } \\
\text { da saúde }\end{array}$ & $\begin{array}{l}\text { Envolvimento em atividades de } \\
\text { recreação diminuído }\end{array}$ & $\begin{array}{l}\text { Desconforto físico } \\
\text { Energia insuficiente }\end{array}$ \\
\hline \multirow{3}{*}{$\begin{array}{l}\text { Atividade/Repouso } \\
\text { Sono/ Repouso }\end{array}$} & Insônia & $\begin{array}{l}\text { Ansiedade } \\
\text { Higiene do sono inadequada } \\
\text { Medo }\end{array}$ \\
\hline & Distúrbio no padrão de sono & $\begin{array}{l}\text { Padrão de sono não } \\
\text { restaurador }\end{array}$ \\
\hline & Privação de sono & Desconforto prolongado \\
\hline $\begin{array}{l}\text { Atividade/Repouso } \\
\text { Atividade/exercício }\end{array}$ & Deambulação prejudicada & Dor \\
\hline $\begin{array}{l}\text { Atividade/Repouso } \\
\text { Equilíbrio de energia }\end{array}$ & Fadiga & Ansiedade \\
\hline \multirow{2}{*}{$\begin{array}{l}\text { Atividade/repouso } \\
\text { Respostas } \\
\text { cardiovasculares/pulmonares }\end{array}$} & Débito cardíaco diminuído & $x$ \\
\hline & Intolerância à atividade & $\begin{array}{l}\text { Desequilíbrio entre a oferta e } \\
\text { a demanda de oxigênio }\end{array}$ \\
\hline \multirow{4}{*}{$\begin{array}{l}\text { Atividade/repouso } \\
\text { Autocuidado }\end{array}$} & Déficit no autocuidado para banho & $\begin{array}{l}\text { Dor } \\
\text { Ansiedade }\end{array}$ \\
\hline & $\begin{array}{l}\text { Déficit no autocuidado para } \\
\text { alimentação }\end{array}$ & \multirow{3}{*}{$\begin{array}{l}\text { Dor } \\
\text { Fadiga }\end{array}$} \\
\hline & $\begin{array}{l}\text { Déficit no autocuidado para higiene } \\
\text { íntima }\end{array}$ & \\
\hline & Déficit no autocuidado para vestir-se & \\
\hline $\begin{array}{l}\text { Percepção/cognição } \\
\text { Cognição }\end{array}$ & Confusão aguda & Dor \\
\hline $\begin{array}{l}\text { Papéis e relacionamentos } \\
\text { Papéis do cuidador }\end{array}$ & Tensão do papel de cuidador & $\begin{array}{l}\text { Condição de saúde instável } \\
\text { Imprevisibilidade do curso da } \\
\text { doença } \\
\text { Dificuldade de acesso à } \\
\text { assistência }\end{array}$ \\
\hline $\begin{array}{l}\text { Sexualidade } \\
\text { Função sexual }\end{array}$ & Padrão de sexualidade ineficaz & $\begin{array}{l}\text { Conflito sobre orientação } \\
\text { sexual }\end{array}$ \\
\hline \multirow{2}{*}{$\begin{array}{l}\text { Enfrentamento/tolerância ao } \\
\text { Estresse } \\
\text { Respostas de enfrentamento }\end{array}$} & Ansiedade & Ameaça à condição atual \\
\hline & Ansiedade relacionada à morte & $\begin{array}{l}\text { Incerteza quanto ao } \\
\text { prognóstico }\end{array}$ \\
\hline $\begin{array}{l}\text { Conforto } \\
\text { Conforto físico }\end{array}$ & Dor aguda & Agente físico lesivo \\
\hline
\end{tabular}

Fonte: Szpalher AS, Batalha MC, 2019. 
Quadro 3-DE potenciais sugeridos ao portador de arritmia.

\begin{tabular}{|c|c|c|}
\hline Domínios/Classes & Diagnósticos de enfermagem potenciais & Condições associadas \\
\hline \multirow{2}{*}{ Nutrição / Hidratação } & Risco de desequilíbrio eletrolítico & Regime de tratamento \\
\hline & Risco de volume de líquidos deficiente & Agente farmacêutico \\
\hline \multirow{5}{*}{$\begin{array}{l}\text { Atividade/repouso } \\
\text { Respostas } \\
\text { cardiovasculares/ } \\
\text { pulmonares }\end{array}$} & Risco de débito cardíaco diminuído & $\begin{array}{l}\text { Alteração na contratilidade / } \\
\text { na frequência cardíaca / no } \\
\text { ritmo cardíaco / no volume } \\
\text { sistólico }\end{array}$ \\
\hline & $\begin{array}{l}\text { Risco de perfusão tissular cardíaca } \\
\text { diminuída }\end{array}$ & Agente farmacêutico \\
\hline & Risco de perfusão tissular cerebral ineficaz & $\begin{array}{l}\text { FA; } \\
\text { Regime de tratamento } \\
\text { Segmento acinético da parede } \\
\text { do ventrículo esquerdo }\end{array}$ \\
\hline & Risco de pressão arterial instável & $\begin{array}{l}\text { Absorção e distribuição rápida } \\
\text { de agente antiarrítmico } \\
\text { Arritmia cardíaca } \\
\text { Desequilíbrio eletrolítico } \\
\text { Reações simpáticas }\end{array}$ \\
\hline & Risco de Intolerância à atividade & Problema circulatório \\
\hline $\begin{array}{l}\text { Percepção/cognição } \\
\text { Cognição }\end{array}$ & Risco de confusão aguda & $\begin{array}{l}\text { Função metabólica } \\
\text { prejudicada }\end{array}$ \\
\hline $\begin{array}{l}\text { Papéis e } \\
\text { relacionamentos } \\
\text { Papéis do cuidador }\end{array}$ & Risco de tensão do papel de cuidador & $\begin{array}{l}\text { Doença crônica } \\
\text { Gravidade da doença }\end{array}$ \\
\hline $\begin{array}{l}\text { Segurança/proteção } \\
\text { Lesão física }\end{array}$ & Risco de quedas & $\begin{array}{l}\text { Agente farmacêutico } \\
\text { Hipotensão ortostática }\end{array}$ \\
\hline
\end{tabular}

Fonte: Szpalher AS, Batalha MC, 2019.

\section{CONSIDERAÇÕES FINAIS}

Observou-se que, mesmo os estudos realizados por enfermeiros apresentando diferentes objetivos, todos convergem em dois pontos: os DE contribuem para o exercício profissional na tomada de decisão; o PE garante a sistematização do cuidado de forma efetiva e resolutiva. Diante da escassa produção, sugeremse estudos que aprofundem o desenvolvimento do PE e planejamento de cuidado direcionado às arritmias prevalentes no âmbito nacional, nos contextos intra e extra-hospitalar, com base na prática baseada em evidências. O desenvolvimento de DE pode promover reflexões acerca do protagonismo do Enfermeiro no manejo da arritmia, ao passo que permite promover a assistência sistematiza vislumbrando, assim, qualidade de assistência, resolubilidade no cuidado e manejo de problemas potenciais e atuais. Ainda se sugere a investigação de diagnósticos em outros domínios da taxonomia, como o domínio 6 - Autopercepção, que não foram abordados neste estudo, visando, assim, a atuação de forma holística.

\section{REFERÊNCIAS}

1. ADRIETTA MP, et al. Plano de alta hospitalar a pacientes com insuficiência cardíaca congestiva. Revista LatinoAmericana de Enfermagem, 2011; 19(6): [08 telas].

2. AHA/ACC/HRS guideline for management of patients with ventricular arrhythmias and the prevention of sudden cardiac death: a report of the American College of Cardiology Foundation/American Heart Association Task Force on Clinical Practice Guidelines and the Heart Rhythm Society. Circulation, 2018;138: 272-391.

3. ALMASHRAFI A, et al. Systematic review of factors influencing length of stay in ICU after adult cardiac surgery. BMC Health Services Research, 2016; 16(318): 2-12. 
4. ALMEIDA DV, et al. Diagnósticos de enfermagem mais frequentes em pacientes internados na Unidade de Terapia Intensiva Coronariana. Revista Arquivos Médicos, 2013; 58: 64-69.

5. ANDRADE MVM, et al. Condutas do enfermeiro nas arritmias cardíacas. Revista de enfermagem UFPE, 2014; 8(3):787-90.

6. NANDA-Internacional: Definições e classificação 2018-2020.11. ed. Porto Alegre: Artmed; 2018; 1187p.

7. BRASIL. Ministério da Saúde. Secretaria de Ciência, Tecnologia e Insumos Estratégicos. Departamento de Ciência e Tecnologia. Diretrizes metodológicas: elaboração de revisão sistemática e metanálise de estudos observacionais comparativos sobre fatores de risco e prognóstico. Brasília: Ministério da Saúde; 2014;132p.

8. CARMO TG. Validação clínica dos diagnósticos de enfermagem risco de recuperação cirúrgica retardada e recuperação cirúrgica retardada em pacientes de cirurgia cardíaca. Tese (Doutorado em Cuidados clínicos) - Escola de Enfermagem Aurora de Afonso Costa. Universidade Federal Fluminense, Niterói, 2018; 181 p.

9. CONSELHO FEDERAL DE ENFERMAGEM. Resolução 358 de 15 de outubro 2009. Brasília - DF.

10. DONATO H, DONATO M. Etapas na Condução de uma Revisão Sistemática. Acta Medicina Portugal, 2019; 32(3): 227-235.

11. GALVÃO PCC, et al. Diagnósticos de enfermagem aplicados a pacientes com insuficiência cardíaca descompensada. Cogitare Enfermagem, 2016; 21(2): 01-08.

12. GONZALEZ B, et al. Sistematização da assistência de enfermagem a um paciente atendido em pronto socorro com arritmia. Salão do conhecimento, 2016; 1-4.

13. LUZIA MF, et al. Nursing Diagnosis Risk for falls: prevalence and clinical profile of hospitalized patients. Revista LatinoAmericana de Enfermagem, 2014; 22(2): 262-268.

14. NOREKVÅL TM, et al. Patient-centred care of patients with ventricular arrhythmias and risk of sudden cardiac death. European Journal of Cardiovascular Nursing, 2017; 16(7): 558-564.

15. NOTTINGHAM F. Diagnosis and treatment of atrial fibrillation in the acute care setting. Journal of the American Academy of Nurse Practitioners, 2010; 22: 280-287.

16. PASSINHO RS, et al. Signs, symptoms and complications of acute myocardial infarction. Revista de enfermagem UFPE, 2018; 12(1): 247-264.

17. REHM J, et al. The relation between different dimensions of alcohol consumption and burden of disease: an overview. Addiction, 2010; 105(5): 817-843.

18. RIENSTRA M, et al. Symptoms and functional status of patients with Atrial Fibrillation. Circulation, 2012; 125: 29332943. 